\title{
Real-time Compliance Control of an Assistive Joint Using QNX Operating System
}

\author{
Shuang $\mathrm{Gu}^{1} \quad$ Cheng-Dong $\mathrm{Wu}^{2} \quad$ Yong Yue $^{1,3} \quad$ Carsten Maple $^{1} \quad$ Da-You Li ${ }^{1} \quad$ Bei-Sheng Liu ${ }^{1}$ \\ ${ }^{1}$ Department of Computer Science and Technology, University of Bedfordshire, Luton LU1 3JU, UK \\ ${ }^{2}$ School of Information Science and Engineering, Northeastern University, Shenyang 110819, China \\ ${ }^{3}$ Department of Computer Science and Software Engineering, Xi'an Jiaotong-Liverpool University, Suzhou 215123, China
}

\begin{abstract}
An assistive robot is a novel service robot, playing an important role in the society. For instance, it can amplify human power not only for the elderly and disabled to recover/rehabilitate their lost/impaired musculoskeletal functions but also for healthy people to perform tasks requiring large forces. Consequently, it is required to consider both accurate position control and human safety, which is the compliance. This paper deals with the robot control compliance problem based on the QNX real-time operating system. Firstly, the mechanical structure of a compliant joint on the assistive robot is designed using Solidworks. Then the parameters of the assistive robot system are identified. The software of robot control includes data acquisition and processing, and control to meet the compliance requirement of the joint control. Finally, a Hogan impedance control experiment is carried out. The experimental results prove the effectiveness of the method proposed.
\end{abstract}

Keywords: Assistive robot, QNX system, compliance control, impedance control, parameters identification.

\section{Introduction}

Assistive robots belong to the kind of human machine to extend the human body function and to amplify human power. They can help the elderly people and handicapped participate in the daily life, such as stroke to complete their normal rehabilitation training so as to achieve an auxiliary healing effect. For a healthy person, an assistive robot can share normal tasks such as bearing of weights, handling of goods and assisting large tasks requiring forces several times of one's own body weight, hence reducing the labour intensity. It can also provide people with force compensation and reduce their energy consumption. For those who have unusual sports behaviour, an assistive robot can provide auxiliary equipment and treatment based on orthopaedic features such as scheduled robot gait behaviour with the user, thus completing the corrective synchronous movements of gait.

For the development of military equipment, soldiers have gradually transferred from handling battlefield weapons into being the core of a comprehensive weapon system. Through the close relationship between an assistive robot and its user, the soldier can distribute the equipment weight to its steel structure, thus transferring the load from the soldier to the robot. This, potentially, can assist soldiers in loading shells, in long distance strikes, in the movement to carry the wounded and strengthen muscles and mobility, speed, endurance, action and load capacity. Assistive robots can also reduce the energy consumption of the soldiers, maximally keeping their fitness, and multiplying combat troops to fight against the enemy.

The assistive robot is a novel service robot, which falls

Manuscript received April 1, 2013; revised July 10, 2013

This work was supported by National High Technology Research and Development Program of China (863 Program) (No. 2011AA040202) and National Nature Science Fundation of China (No. 51005008). into the special robot category. An assistive robot represents a high integration of robotics, ergonomics, control engineering, sensor technology, communications and signal processing. The assistive robot is designed for normal human limb power augmentation, and it has potential applications for elderly people, handicapped, succour, care-worker, soldier and fireman. The assistive system consists of a fundamental exoskeleton, actuator, controller and many sensors for obtaining user motion information, and becomes a humanised system. There has been increased attention to research on assistive robots ${ }^{[1-13]}$, and well-known systems include Berkeley lower extremity exoskeleton (BLEEX) ${ }^{[14]}$ of Berkeley University and the Japan University of Tsukuba hybrid assistive limb (HAL) system ${ }^{[15]}$. Consequently, it is required to consider both accurate position control and human safety, which is the compliance. An assistive robot frequently interacts with humans and there is a demand for a breakthrough of technologies underpinning wearable assistive devices.

In order to achieve the robot compliance, a range of application methods have been proposed, including passive compliance arithmetic and active compliance arithmetic. The passive compliance is controlled by an assistive compliant device whilst the active compliance is controlled by a method called force feedback control.

Passive robot compliance between the environment and human effect can use mechanical devices with absorbed or stored energy, such as spring and damping, to produce the natural obedience. It has played an important role in position error compensation for vibration energy absorption and increasing processing compliance.

In assistive robot applications, there exist many different methods such as series elastic actuation (SEA), pneumatic artificial muscle device and various spring devices. For instance, Tokyo Industry University ${ }^{[16]}$ and German Centre $^{[17]}$ respectively used variable stiffness joint struc- 
tures; however, the compliance joint stiffness is decided by the structure, and it is not flexible for independent control. Korea Technology University ${ }^{[18]}$ employed a two-concentric magnet circle method to achieve the controllable stiffness, but this method has a low accuracy and the control of the two-concentric magnet circle structure requires extra coordination. One of the most famous passive compliance devices, developed by Massachusetts Institute Technology (MIT) Laboratory ${ }^{[19]}$ Draper is called remote centre compliance (RCC). It consists of six springs to perform compliant movements around a remote centre. The RCC is widely used in robotic assembling.

The Israel Brussels research team built two kinds of walking robot: a pleated pneumatic artificial muscles (PPAM) driven robot called "Lucy" and a mechanically adjustable compliance and controllable equilibrium position actuator (MACCEPA $)^{[20]}$ multi-stiffness equilibrium position driven robot called "Veronica". The team also conducted research on flexible joint arms and developed a two-link robot arm driven by PPAM. The assistive leg developed by Vrije University ${ }^{[21]}$ and the hybrid-actuated robot arm developed by Stanford University ${ }^{[22]}$ use pneumatic artificial muscles to actuate the flexible joints. The artificial driver can be controlled by changing the air pressure of a pneumatic driver. The actuation of the artificial muscle is similar to human muscles so it increases the flexibility of the joint movement. However the power supply of the artificial muscle is too heavy for a wearable device and the noise generated by the actuator could also limit the application of the artificial muscles.

Rahman et al. ${ }^{[23]}$ developed a 4-degree-of-freedom (DoF) exoskeleton robot (called ExoRob) corresponding to human shoulder and elbow movements to provide effective rehabilitation for physically disabled individuals with impaired upper-limb functions. The control of ExoRob is implemented by a non-linear sliding mode technique. Experiment results show that ExoRob is able to provide effective rehabilitation for the shoulder and elbow joint movements. Vitiello et al. ${ }^{[24]}$ presented a novel exoskeleton NEUROExos for elbow rehabilitation. NEUROExos uses double-shelled links and a compliant antagonistic actuation system for providing gently torque transmission to the human joint antagonistic actuation system allows independent control of the joint position and stiffness which enables the joint stiffness to be changed in a wide range.

There are a number of active compliance methods such as impedance control, adaptive impedance control, force/position hybrid control and intelligence control, among which impedance control is more mature and simpler to implement, for example, robot applications.

In 1985, Hogan ${ }^{[25]}$ systematically expounded the concept of impedance and put forward a dynamics model for robot impedance control. Hogan impedance control method makes a difference in the development of impedance control strategy. The best way of force control is to treat the force and position as two different variables and control them at the same time. Theoretically, the force space and the position space of a robot are orthogonal to each other. The proposed algorithm uses a diagonal matrix to separate the robot motion space into two sub-spaces, which are force space and position space. The normal line of the target object surface is the force space where the force control is applied. The tangent line of the target object surface is the position space where the position control is applied.

Qiu and $\operatorname{Tan}^{[26]}$ proposed a hybrid force/position controlwhich uses a Jacobi matrix to allocate a motion to the robot's joint position/force changes. The robot is able to be controlled in terms of position and force at the same time. Li et al. ${ }^{[27]}$ used an adaptive method to reduce the large force error in impendence control. The reference position is adjusted according to the force error so the changes of the environment can be tracked. This method is robust to uncertainties raised by unstructured/changing environments and its short computation time can fulfil the requirement of real-time control. The position/force control often requires an exact modelling of the environment and accurate sensor readings. These sometimes cannot be achieved in the daily task implementation.

Lee et al. ${ }^{[28]}$ developed a control scheme of an assistive robotic cart that helps a user easily transport objects of various weights. Pre-determined impedance between the user and the cart is regulated to generate an assist force using the user's input force and the velocity of the cart. $\mathrm{Su}$ et al. ${ }^{[29]}$ developed an exploratory and perceptual algorithm for a mechatronic robotic system equipped with liquid-filled, biomimetic tactile sensors (BioTac). The tactile sensors can be used to measure compliance of objects. The distribution of force on the fingertip is measured by the electrical resistance of the conductive liquid. These signals provide closed-loop control of exploratory movements, while the distributions of skin deformations are used to estimate material properties of objects.

The task of this paper is to design a kind of assistive robot that can assist either a person's upper limbs or lower limbs (Fig. 1). As a service robot, it frequently interacts with humans and it could help humans complete the auxiliary function but does not hurt them. Therefore, our motivation is to develop an assistive robot compliant actuation. The experiment equipment of assistive robot's compliant joint was designed using Solidworks. Key technologies of the robot system are studied and presented, including safety design, characteristics of the force sensor, and the influence toward the control precision.

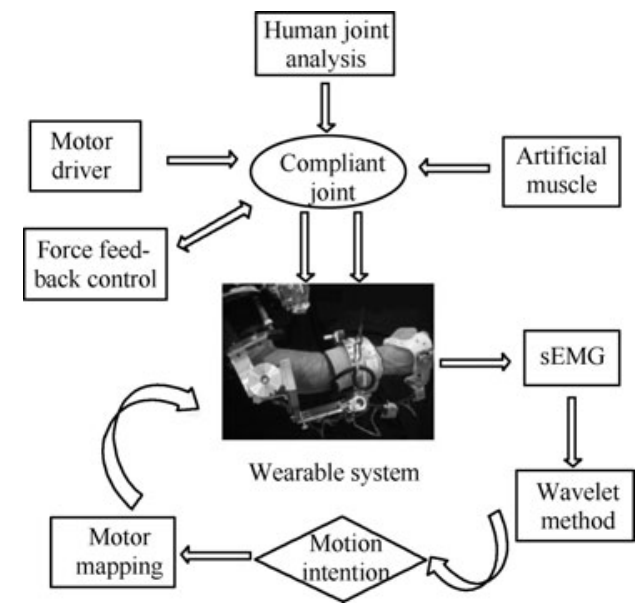

Fig. 1 The structure of the wearable system 
In this research, an experimental platform for assistive robot control is established as one of the most important parts in the wearable system. An experiment for identifying the user's intension obtained by surface electromyography (sEMG) of the compliant joint movement is performed. Much research has been done on a computer system without a suitable real-time operating system. This work is based on the QNX operating system which is a real-time and multi-tasking system. Based on the QNX real-time platform, the robot system is located in the control loop. The control model actualises all kinds of modes such as force signal model and position initialisation model.

\section{Assistive robot control system}

The assistive robot in the experiment system shown in Fig. 2 has three parts: EMG system, QNX control system and the joint actuator.

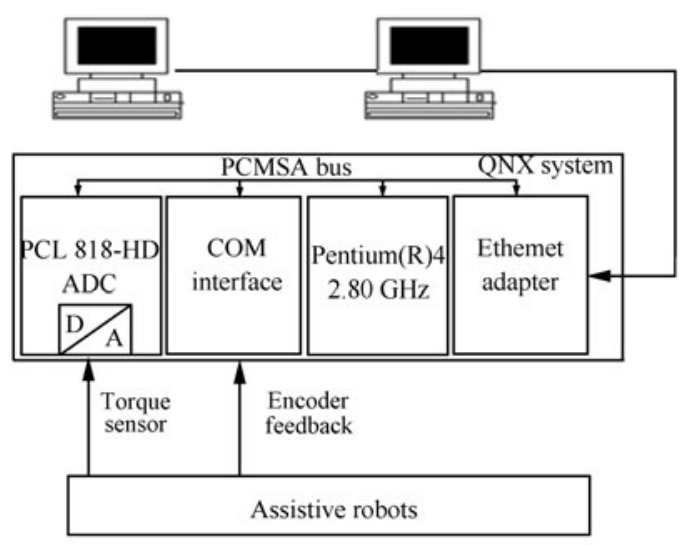

Fig.2 QNX system-based experimental platform for the assistive robot's compliant joint

The structure of the system is designed based on the standard close-loop control including the robot mechanism system, QNX system (host computer), motor driver circuits and a torque sensor. The operating system of the PC is the QNX which enables the measurement, control and monitoring in real time. Real-time processing and communications using the network are required for the control purpose. A LAN card which has an $11 \mathrm{Mbps}$ transmission rate and an $\mathrm{A} / \mathrm{D}$ (analogue to digital) converter card which has 16 channels ( 8 bit resolution) input are used. Sensor readings are sent to an $\mathrm{AD}$ convertor of the QNX system. Control signals are sent to the electronically communicated (EC) motor through a COM interface. The QNX system communicates with the PCs through Ethernet. The QNX system enables the measure, control and system monitoring in real time.

\section{Hardware of assistive robot}

The compliant actuation of the assistive robot is compared with the theoretic analysis of the human body's joint which is a passive compliance method. A motor controlled compliant joint with a torque sensor is developed.

The schema of the assistive robot's actuated joint system is shown in Fig. 3, which is for an arm joint with one degree of freedom. This is an experimental device for an assistive robot where restriction is imposed at the joint mobile angle to ensure user safety. Aluminium alloy and steel are used as the materials of the frame in consideration of weight constraint. The actuator system of the assistive robot provides the assistive force for the arm joint. The actuator consists of an EC-motor and harmonic drive to generate the torque needed at the joint. The actuator using a harmonic drive is assembled compactly with a large reduction gear ratio and smooth drive.

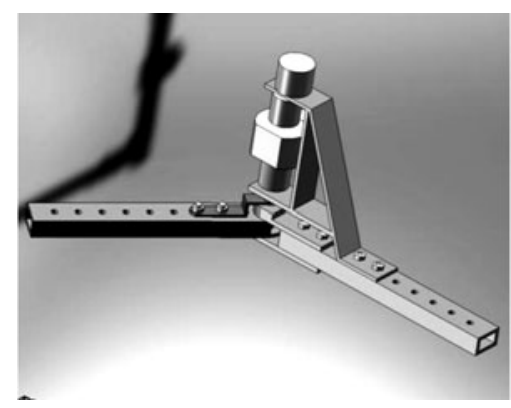

Fig. 3 The experimental equipment of assistive robot

\subsection{Peripheral component interconnection}

Peripheral component interconnection (PCI) is the external device. A PCI bus is located in the interconnection of microprocessor with the standard local bus to expand the bus structure. PCI is used to bridge the road connecting with the local bus PCI but not directly connected with the microprocessor. In addition, the PCI has good compatibility suitable for all kinds of models, high-efficiency, obligate space for development, etc.

The data acquisition module can also display data changes in real-time. In addition, the acquired data will be saved in a specific file for further analysis.

\subsection{Motor controller}

The control system uses a FAULHABER motor as the driver device. The motor controller is designed for EC micro-motor MCD2805 and with embedded encoder IE2512. In the slow-speed model, the precision of the motor position can achieve $0.18^{\circ}$. This can fulfil the requirements of the robot arm control. MCD2805 is developed from a powerful 16 bits microprocessor. The controller also has an excellent digital filter.

\subsection{Data acquisition card}

The data acquisition card adapts a PCL 818-HD A/D board (shown in Fig. 4), which contains a $1 \mathrm{~K}$ sampling first-in-first-out (FIFO) buffer for fast data transmission. It is suitable for signal acquisition and measurement. The PCL 818-HD has 16-bit single input or 8-bit difference analogue input, 12-bit differential A/D sampling rate, $100 \mathrm{kHz}$, 2 function switches and 11 jumpers. The analogue input ranges can be chosen by the software. The available ranges of dual polarity are $\pm 1.625 \mathrm{~V}, \pm 1.25 \mathrm{~V}, \pm 1.625 \mathrm{~V}, \pm 2.5 \mathrm{~V}$, $\pm 5 \mathrm{~V}, \pm 10 \mathrm{~V}$ and the available ranges of single polarity are 0-125 V, 0-2.5 V, 0-5 V, and 0-10 V. 


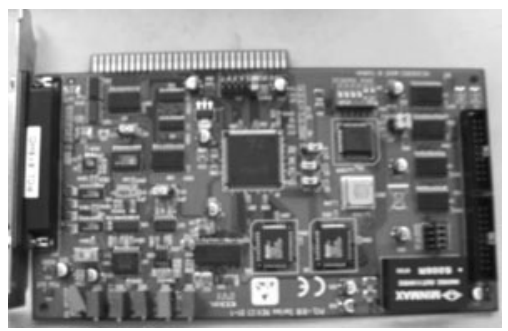

Fig. 4 PCL 818-HD data acquisition card

\subsection{Sensor device}

The robot needs to be monitored in real-time and the requirement of joint torque accuracy needs to be achieved by experiments. The platform used in the experiments-T22 torque sensor model (1-dimensional) is provided by HBM Company. The measuring range of the torque sensor is from $+5 \mathrm{~N} \cdot \mathrm{m}$ to $-5 \mathrm{~N} \cdot \mathrm{m}$ with a precision of $5 \%$. The supply voltage is $24 \mathrm{~V}$ electronically communicated (AC) and the output range is from $-5 \mathrm{~V}$ to $+5 \mathrm{~V}$. The torque signal is sampled by an A/D card and sent to the PC.

\section{QNX based software system}

\subsection{Operating system}

$\mathrm{QNX}^{[30]}$ developed by the Canadian QNX Software Systems Company is a multi-task, distributed and embedded real-time operating system. The micro kernel of QNX is of small size and the process switch-time is very short according to the POSIX protocol API, making it an interconnected system and convenient to implant for the UNIX/LINUX system. Therefore, QNX has been widely adopted in embedded systems, robot engineering and industrial control, aerospace, etc. QNX for the Windows development environment is in Fig. 5 .

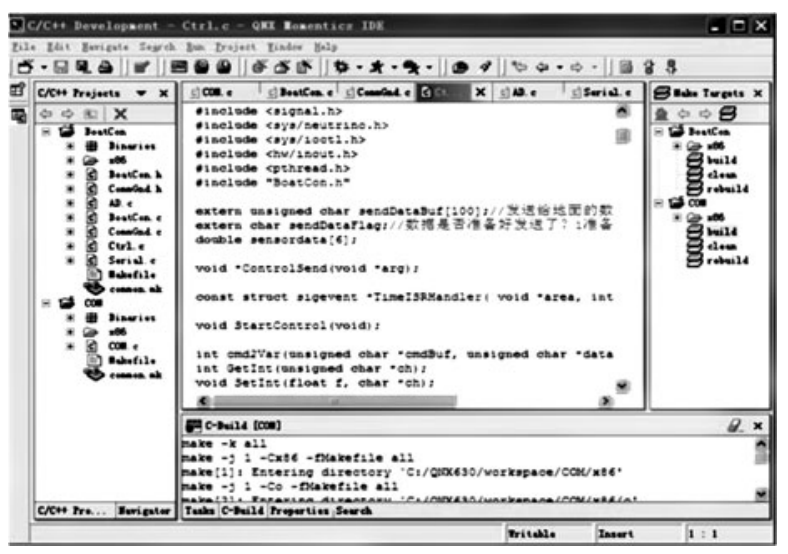

Fig.5 QNX for Windows development environment

\subsubsection{System structure}

The micro kernel of QNX is of small size and the minimum configuration only takes $10 \mathrm{~KB}$ of memory. The QNX operating system offers four core services: process scheduling, inter-process communication, underlying network communication and interrupt handling; especially, the process operates in the address space independently.

\subsubsection{Scheduling strategy}

QNX provides the POSIX.1b standard process scheduling. Within QNX, the scheduling has 32 priorities from 0 to 31 based on a first-come first-served priority policy for context switch in order to satisfy the waiting requirements for the CPU time.

\subsubsection{System services}

The system services include resource management, equipment management, graphic/window support, TCP/IP and distributed management:

1) Resource management: It includes file system and device management, full POSIX.1 and the UNIX syntax for POSIX file system. It supports multiple file system running at the same time, multiple flash memory devices embedded file system and multiple file servers (such as Windows and LAN Manager) transparent access to the SMB file system, DOS file system and CD-ROM file system.

2) Equipment management: It provides the interface service between the process and terminal equipment.

3) Graphic/window support: It includes QNX Windows, $\mathrm{X}$ Window System for QNX and Windows remote graphics connection.

4) Performance and QNX type fault-tolerant FLEET.

\subsection{Open system}

The open system includes the POIX compatibility of QNX, CPU supporters, various buses and peripheral equipment:

1) The POIX compatibility of QNX: QNX can provide the characteristic of UNIX compiler, debuggers, X Windows and TCP/IP.

2) Various CPU supporters: AMD ElanSC300/310/400/ 410, Am386 DE/SE, Cyrix MediaGX, x86 processor, Pentium series and STPC of STMicroelectronics.

3) Various buses: Compact PCI, EISA, ISA, MPE (RadiSys), STDS, STDS, 32, PC/104, PC/104-Plus, PCI, PCMCIA, VESA and VME.

4) Various peripheral equipment: SCSI devices, IDE/ EIDE drives, $10 \mathrm{M} / 100 \mathrm{M}$ Ethernet card, Token Ring network interface, FDDI interface, PCMCIA device, memory, etc.

Since the data acquisition and robot control are required in real-time, the PC operating system must have a real-time kernel. Due to its characteristics, this research has chosen QNX as the operating system platform.

\subsection{Software structure}

This software is written in the $\mathrm{C}$ programming language within the PC operating software. The architecture of the software programming uses a main program + task + interrupt structure. The main function includes data measured from the torque sensor, control motor, communication between the host PC and the second PC. The software includes the main program module, initialisation module, motor serial interface communication module, network communication module, software timer interrupt module and serial interface interrupt module. The pseudo-code of the main program is shown in Fig. 6 . 


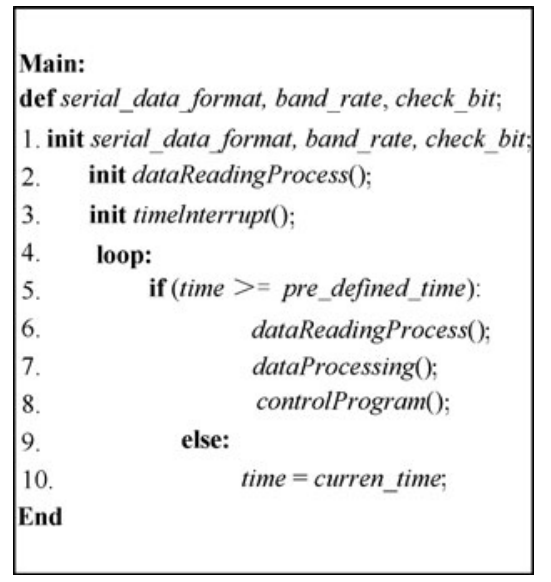

Fig. 6 Pseudo-code of the main program

\subsection{Analysis of software function}

\subsubsection{Data acquisition}

The data is transmitted through the motor serial interface. Therefore, the serial interface needs to be set when the program is initialised including the serial data format, band rate, and check bit. The function is accomplished through a serial interruption. The pseudo-code of the data acquisition and the interrupt service routine is shown in Fig. 7. The interruption consists of an interrupt service routine (ISR) and interrupt task.

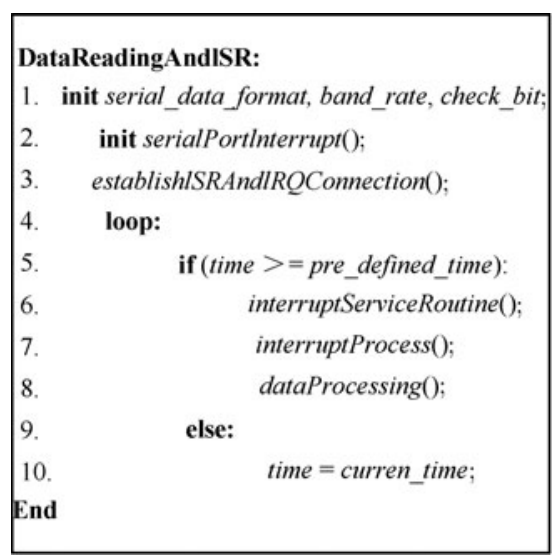

Fig. 7 Pseudo-code of data acquisition and interrupt service routine (ISR)

ISR bound is placed to all the IRQx ports in order to call ISR accordingly when the IRQx is interrupted. Then ISR calls the interruption task and data processing.

\subsection{Network communication}

Network communications are established between the upper PC and lower PC so that the upper PC can read the real-time state of the robot and send the instruction to the lower PC. This research uses a TCP/IP network communication because of its high-speed and reliability for network communication. TCP is a connection-oriented and reliable data transmission. Therefore, the connection in the TCP protocol must be built before data exchange takes place between the sender and receiver. Then the data in the upper
PC can be transferred to the lower PC to control the assistive robot. In the meantime, the information about the statement of the assistive robot is achieved by the upper $\mathrm{PC}$ through the lower PC in real-time. Therefore, the upper $\mathrm{PC}$ is used as the user interface and the lower-PC as a server.

The main design steps of the server process are as follows:

1) Create TCP port socket: socket();

2) Bind service port: $\operatorname{bind}()$;

3) Set the port socket into listening socket: listen();

4) Accept new connection: accept();

5) Receive the information from the sender: $\operatorname{recv}()$.

The main design steps of the server process are as follows:

1) Create TCP port socket: socket();

2) Designate the IP address and port of server;

3) Establish the connection to the server: connect();

4)Call the function select() for a time;

5) Send messages to the specified address and port: write ()

\section{Establishment of dynamic robot model}

The dynamic model of a manipulator provides a description of the relationship between the joint actuator torques and the motion of the structure ${ }^{[31,32]}$. There are two methods for derivation of the equations of motion of a manipulator in the joint space. The first method is based on the Lagrange formulation and is conceptually simple and systematic. The second method is based on the Newton-Euler formulation and allows obtaining the model in a recursive form; it is computationally more efficient since it exploits typically the open structure of the manipulator's kinematic chain.

With Lagrange formulation, the equations of motion can be derived in a systematic way independently of the reference coordinate frame. Once a set of variables $q_{i}$, termed generalised coordinates, are chosen which effectively describe the link positions of an $n$-degree-of-mobility manipulator, $d_{1}$ and $d_{2}$ are the distances from the joints to the center of mass for each link, as shown in Fig. 8. The Lagrange of the mechanical system can be defined as a function of the generalised coordinates:

$$
L=K-P
$$

where $K$ and $P$ are respectively the total kinetic energy and potential energy of the system.

The Lagrange's equations are expressed by

$$
F_{i}+\frac{\mathrm{d}}{\mathrm{d} t}\left[\frac{\partial L}{\partial \dot{q}_{l}}-\frac{\partial L}{\partial q_{i}}\right], \quad i=1,2,3, \cdots, n
$$

where $F_{i}$ is the generalised force associated with the generalised coordinate $q_{i}$.

Therefore, the dynamics of a two-link planar robot is

$$
I(\theta) \ddot{\theta}+C(\theta, \dot{\theta}) \dot{\theta}+g(\theta)=\tau .
$$

In this equation, $\theta, \dot{\theta}$ and $\ddot{\theta}$ are vectors of the generalised position, velocity and acceleration variables, respectively, 
and $\tau$ is a vector of the generalised torque. $I$ is the generalised inertia matrix. From the equation, $I(\theta)$ depends on $\theta$. Likewise, $C$ is the generalised bias force, written as $C(\theta, \dot{\theta})$ which depends on both $\theta$ and $\dot{\theta}$.

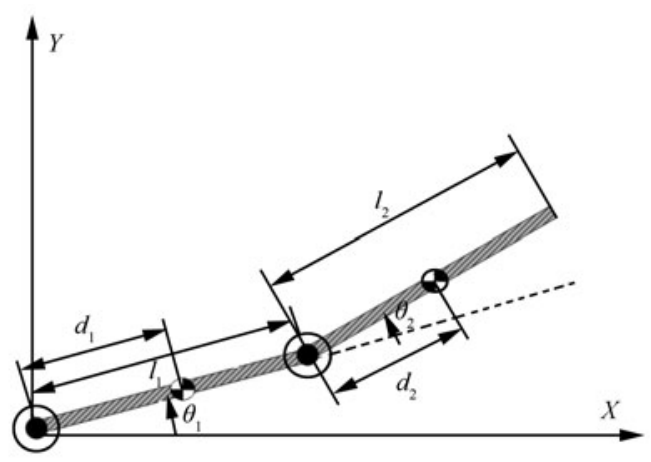

Fig. 8 Two-link planar manipulator

The torque vector is $\tau=\left[\tau_{1} / \tau_{2}\right]$ and the joint angle vector is $\tau=\left[\tau_{1} / \tau_{2}\right]$. The inertia matrix is

$$
\begin{aligned}
& I(\theta)= \\
& {\left[\begin{array}{cc}
\left(m_{1}+m_{2}\right) d_{1}^{2}+m_{2} d_{2}^{2}+2 m_{2} d_{1} d_{2} \cos \theta_{2} & m_{2} d_{2}^{2}+m_{2} d_{1} d_{2} \cos \theta_{2} \\
m_{2} d_{2}^{2}+m_{2} d_{1} d_{2} \cos \theta_{2} & m_{2} d_{2}
\end{array}\right] .}
\end{aligned}
$$

The Coriolis centrifugal force matrix is:

$$
\begin{aligned}
& c(\theta, \dot{\theta})= \\
& {\left[\begin{array}{c}
2 m_{2} d_{1} d_{2} \sin \theta_{2} \cdot \dot{\theta}_{1} \dot{\theta}_{2}+m_{2} d_{1} d_{2} \sin \theta_{2} \cdot \dot{\theta}_{2}^{2} \\
m_{2} d_{1} d_{2} \sin \theta_{2} \cdot \dot{\theta}_{1}^{2}
\end{array}\right] .}
\end{aligned}
$$

The gravitational matrix is

$$
\begin{aligned}
& g(\theta)= \\
& {\left[\begin{array}{c}
\left(m_{1}+m_{2}\right) g d_{1} \cos \theta_{1}+m_{2} g d_{2} \cos \left(\theta_{1}+\theta_{2}\right) \\
m_{2} g d_{2} d_{2} \cos \left(\theta_{1}+\theta_{2}\right)
\end{array}\right] .}
\end{aligned}
$$

The common dynamic equations do not contain all the forces of the operation arm, for example, the friction.

Considering the friction, the friction model is as follows.

1) Coulomb friction

$$
\tau_{\text {friction } 1}=\operatorname{csgn}(\dot{\theta})
$$

where $c$ is the Coulomb friction constant, and the signal of $c$ depends on the velocity of the joint.

When $\dot{\theta}=0, c=1$, which is called the static friction coefficient.

When $\dot{\theta} \neq 0, c<1$, which is called the dynamic friction coefficient.

2) Viscous fiction

$$
\tau_{\text {friction } 2}=\nu \dot{\theta}
$$

where $v$ is the viscous friction coefficient.

Therefore, the friction model equation is

$$
\tau_{\text {fiction }}=\tau_{\text {friction } 1}+\tau_{\text {fiction } 2}=\operatorname{csgn}(\dot{\theta})+\nu \dot{\theta} .
$$

Meanwhile, the dynamics equation of a two-link planar robot considering friction is

$$
\begin{array}{r}
I \ddot{\theta}+\tau_{\text {fiction }}+C(\theta, \dot{\theta}) \dot{\theta}+G(\dot{\theta})=\tau \\
I \ddot{\theta}+v \dot{\theta}+\operatorname{sggn}(\dot{\theta})+C(\theta, \dot{\theta}) \dot{\theta}+G(\dot{\theta})=\tau .
\end{array}
$$

The dynamics equation of a one-link planar robot is

$$
\begin{gathered}
I \ddot{\theta}+v \dot{\theta}+c \operatorname{sgn}(\dot{\theta})+g(\dot{\theta})=\tau \\
g(\theta)=\frac{1}{2} m g d \cos \theta .
\end{gathered}
$$

\section{Experiment analyses}

\subsection{Identification of parameters}

In order to design the controller, the system model needs to be identified. As a consequence, an experiment is designed and carried out to identify parameters of the singlejoint assistive robot. During the experiment, the assistive robot can move according to the operator's intention. The experimental results are shown in Fig. 9.
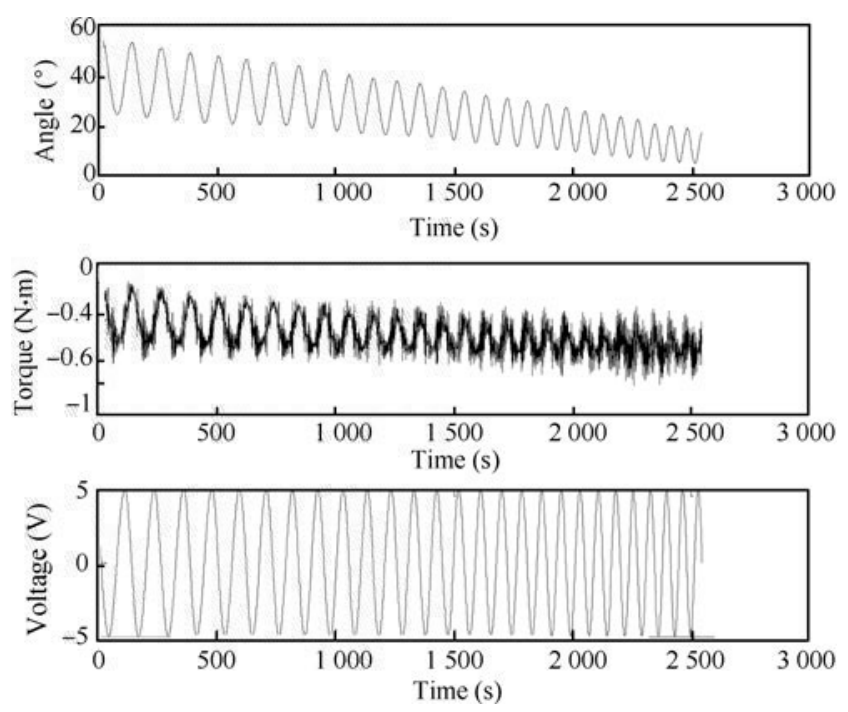

Fig. 9 Experimental results (angle of joint signal, torque sensor output signal and voltage signal)

Based on the test data, the least-square method is used to identify the dynamics equation parameters of the assistive robot. The model parameters identified are as follows:

$$
I=0.1647, v=-0.3054, c=-0.0093 .
$$

The motor model parameter identification (torque transformation to voltage model) is as follows:

$$
\ddot{\tau}+k_{1} \dot{\tau}+k_{2} \tau=b_{1} \dot{u}+b_{2} u
$$

where $k_{1}=0.0676, k_{2}=-0.3138, b_{1}=0.0015, b_{2}=0.0009$ represent respectively identification parameters; $\tau$ represents joint torque; $u$ represents input voltage.

\subsection{Hogan impedance control experiment}

The application in which a robot's end-effecter interacts with the environment requires a suitable compliant 
behaviour to be implemented. A well-established framework to manage this task is given by impedance control to gain the desired implementation. Impedance control is inherently a model-based approach. According to the target impedance, position-based impedance control is a proven method which is stable and robust. This research makes use of Hogan impedance control based on position control for the assistive robot in which positions are determined while forces are measured.

The aim of an impedance controller is to establish a massdamper-spring relationship between the position and the force. The desired impedance equation of the assistive robot can be represented by

$$
M_{d}\left(\ddot{X}_{r}-\ddot{X}\right)+B_{d}\left(\dot{X}_{r}-\dot{X}\right)+K_{d}\left(X_{r}-X\right)=F_{d}
$$

or

$$
F_{d}=\left(M_{d} s^{2}+B_{d} s+K_{d}\right) X_{e}=Z X_{e}
$$

where $K_{d}, B_{d}, M_{d}$ represent the desired inertia, damping and stiffness of the system, respectively; $F_{d}$ represents the force acting on the end-effecter; $X, \dot{X}, \ddot{X}$ represent the acceleration, velocity and position of the end-effecter, respectively; $X_{r}, \dot{X}_{r}, \ddot{X}_{r}$ represent the reference acceleration, velocity and position of the end-effecter, respectively; and $X_{e}$ represents the position error. Fig. 10 gives an overview of the controller structure implemented on the assistive robot where there is a position control in the outer loop and a force control in the inner loop.

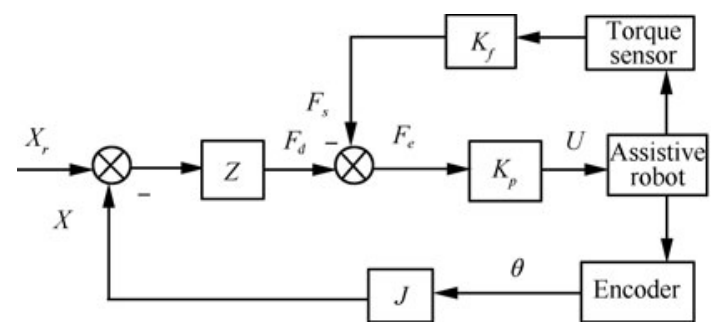

Fig. 10 Impedance control structure

Firstly, the robot is expected to move in a free space without force feedback measured by the sensor, i.e., $F_{s}=$ $0, F_{e}=F_{d}$. The expected position of the joint acts as the position signal input directly.

$$
U=F_{e} K_{p}=X_{e} Z K_{p}=\left(X_{r}-X\right) Z K_{p} .
$$

Secondly, when the robot is in the restricted environment, in other words, the end-effecter of the robot is forced by the manipulator $\left(F_{s} \neq 0\right)$, the force loop is closed. At the same time, position error $X_{e}$ is generated by force $F_{d}$. $F_{d}$ is compared with the feedback force signal for error correction. With the position error parameter increasing, the force of the robot end-effecter increases as well. Hence, the target impedance parameters $\left(K_{d}, B_{d}, M_{d}\right)$ and the force feedback ratio $K_{f}$ can be regulated to control the relationship between the robot end-effecter and environment. This is the force property of the manipulator, and the equation is defined as follows.

$$
U=\left(F_{d}-F_{s}\right) K_{P}=\left(X_{e} Z-F_{s}\right) K_{p}
$$

where $Z$ represents impedance parameter, and $K_{p}$ represents the parameter of the force close loop controller.

The experiment equipment for the assistive robot's compliant joint is shown in Fig. 11. During the process of experiment, the robot produces a resistant force when the manipulator pushes the robot. Gradually the robot is back to the balance position. The property of the robot is like a spring and the resistant force can be changed by the regulator parameters. Meanwhile, the moveable range of the joint becomes wider.

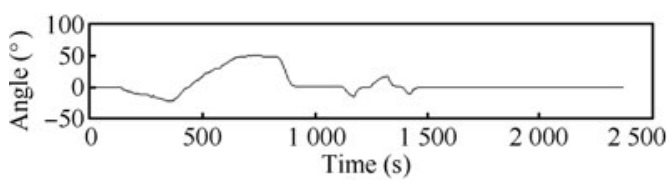

(a)

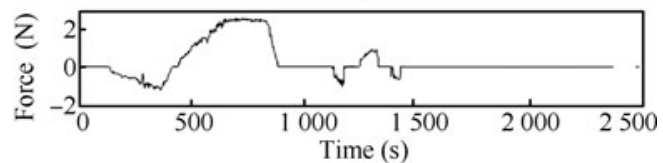

(b)

Fig. 11 Experimental results

In order to test our algorithm, the force feedback ratio is set to $K_{f}=-5$ and inertia is set to $K_{d}=80$, the joint's position response error and the corresponding force response in the direction of the joint movement are illustrated in Fig. 11, where (a) is concerned with the force feedback measured by the sensor in the direction of the joint; (b) is concerned with the position error of the joint. From Fig. 11, it can be seen that the trend of the joint response error is similar to the trend of the force signal feedback; in other words, the force control can be converted into the robot's position control. Furthermore, the position error is close to zero as the force disappears. The results show the effectiveness of the proposed impedance control method for the compliant joint.

\section{Conclusions}

This paper has presented the design and implementation of an experimental platform for assistive robot compliant actuation. An experiment is carried out on this platform for assistive robot control with the sensor and controller integrated. The control system software of the assistive robot is designed based on the QNX real-time operation system and an experiment is accomplished to identify parameters for the assistive robot dynamic equation model. Experimental results have demonstrated the effectiveness and advancement of the control system as well as the assistive robot experimental platform.

\section{References}

[1] M. Zinn, O. Khatib, B. Roth, J. K. Salisbury. Playing it safe - A new actuation concept for human-friendly robot design. IEEE Robotics \& Automation Magazine, vol.11, no. 2, pp. 12-21, 2004.

[2] M. Zinn, O. Khatib, B. Roth. A new actuation approach for human friendly robot design. In Proceedings of 2004 IEEE International Conference on Robotics and Automa- 
tion (ICRA'04), IEEE, New Orleans, LA, USA, vol.1, pp. 249-254, 2004.

[3] S. A. Migliore, E. A. Brown, S. P. DeWeerth. Biologically inspired joint stiffness control. In Proceedings of 2005 IEEE International Conference on Robotics and Automation, IEEE, Atlanta, Georgia, pp. 4508-4513, 2005.

[4] B. J. Makinson. Research and Development Prototype for Machine Augmentation of Human Strength and Endurance, Hardiman I Project, General Electric Report S-71-1056, Schenectady, NY, USA, 1971.

[5] D. Vischer, O. Khatib. Design and development of highperformance torque-controlled joints. IEEE Transactions on Robotics and Automation, vol.11, no. 4, pp. 537-544, 1995.

[6] G. A. Pratt, M. Williamson. Series elastic actuators. In Proceedings of IEEE/RSJ International Conference on Intelligent Robot Systems, IEEE, Pittsburgh, PA, USA, pp. 399406, 1995.

[7] S. Wolf, G. Hirzinger. A new variable stiffness design: matching requirements of the next robot generation. In Proceedings of 2008 IEEE International Conference on Robotics and Automation, IEEE, Pasadena, USA, pp. 1741-1746, 2008.

[8] T. Nakamura, K. Saito, Z. D. Wang, K. Kosuge. Control of model-based wearable anti-gravity muscles support system for standing up motion. In Proceedings of 2005 IEEE/ASME International Conference on Advanced Intelligent Mechatronics, IEEE, Monterey, CA, USA, pp. 564569, 2005.

[9] X. G. Zhao, S. Gu, C. D. Wu, B. Yang, J. D. Han. QNX based control system of assistive robot. Chinese Journal of Scientific Instrument, vol. 30, no. 10, pp. 322-325, 2009. (in Chinese)

[10] S. Gu, Y. Yue, C. Maple, C. Wu. QNX based on human movement intention control research on assistive robot. In Proceedings of the 16th International Conference on $\mathrm{Au}$ tomation and Computing. University of Birmingham, UK, 2010.

[11] S. Gu, Y. Yue, C. Maple, C. D. Wu, B. S. Liu. Classification of multi-channels SEMG signals using wavelet and neural networks on assistive robot. In Proceedings of the 10th IEEE International Conference on Industrial Informatics (INDIN), IEEE, Beijing, China, pp. 1158-1163, 2012.

[12] S. Gu, Y. Yue, C. Wu, C. Maple, D. Li, B. Liu. sEMG based intention identification of human body movement research on assistive robot. Romanian Academy Journal Technology Science, vol. 57, no. 2-3, pp. 217-233, 2012.

[13] S. S. Groothuis, S. Stramigioli, R. Carloni. Lending a helping hand: Toward novel assistive robotic arms. IEEE Robotics \& Automation Magazine, vol. 20, no. 1, pp. 20-29, 2013.

[14] A. Zoss, H. Kazerooni, A. Chu. On the mechanical design of the Berkeley lower extremity exoskeleton (BLEEX). In Proceedings of IEEE/IRSJ International Conference on Intelligent Robots and Systems (IROS 2005), IEEE, Tokyo Big Sight, Japan, pp. 3132-3139, 2005.

[15] Y. Sankai. Leading edge of cybernics: Robot suit HAL. In Proceedings of International Joint Conference on SICEICASE, IEEE, Busan Exhibition \& Convention Center BEXCO, Busan, Korea, pp. P-1-P-2, 2006.

[16] M. Okada, S. Kino. Torque transmission mechanism with nonlinear passive stiffness using mechanical singularity. In Proceedings of 2008 IEEE International Conference on Robotics and Automation, IEEE, Pasadena, CA, USA, pp. 1735-1740, 2008.
[17] S. Wolf, G. Hirzinger. A new variable stiffness design: Matching requirements of the next robot generation. In Proceedings of 2008 IEEE International Conference on Robotics and Automation, IEEE, Pasadena, CA, USA, pp. 1741-1746, 2008.

[18] J. Choi, S. Park, W. Lee, S. Kang. Design of a robot joint with variable stiffness. In Proceedings of 2008 IEEE International Conference on Robotics and Automation, IEEE, Pasadena, CA, USA, pp. 1760-1765, 2008.

[19] M. T. Mason. Compliance and Force Control for Computer Controlled Manipulators, Ph.D. dissertation, MIT, Massachusetts, USA, 1979.

[20] R. Van Ham, B. Vanderborght, M. V. Damme, B. Verrelst, D. Lefeber. MACCEPA: The mechanically adjustable compliance and controllable equilibrium position actuator for controlled passive walking. In Proceedings 2006 IEEE International Conference on Robotics and Automation (ICRA 2006), IEEE, Orlando, FL, USA, pp. 2195-2200, 2006.

[21] B. Vanderborght, B. Verrelst, R. Van Ham, D. Lefeber. Controlling a bipedal walking robot actuated by pleated pneumatic artificial muscles. Robotica, vol. 24, no. 4 pp. 401-410, 2006.

[22] D. Shin, I. Sardellitti, O. Khatib. A hybrid actuation approach for human-friendly robot design. In Proceedings of 2008 IEEE International Conference on Robotics and $\mathrm{Au}$ tomation, IEEE, Pasadena, CA, USA, pp. 1747-1752, 2008.

[23] M. H. Rahman, T. K. Ouimet, M. Saad, J. P. Kenné, P. S. Archambault. Development of a 4 DoFs exoskeleton robot for passive arm movement assistance. International Journal of Mechatronics and Automation, vol. 2, no. 1, pp.34-50, 2012

[24] N. Vitiello, T. Lenzi, S. Roccella, S. M. M. de Rossi, E. Cattin, F. Giovacchini, F. Vecchi, M. C. Carrozza. NEUROExos: A powered elbow exoskeleton for physical rehabilitation. IEEE Transactions on Robotics, vol.29, no. 1, pp. 220-235, 2013.

[25] N. Hogan. Impedance control: An approach to manipulation: Parts I, II and III. Journal of Dynamic Systems, Measurement, and Control, vol. 107, no. 1, pp. 1-24, 1985.

[26] Z. C. Qiu, D. L. Tan. On acceleration sensor-based feedback control for contact force of the flexible joint manipulator. Chinese Journal of Mechanical Engineering, vol. 38, no. 10, pp. 37-41, 2002. (in Chinese)

[27] J. Li, Q. Wei, W. S. Chang, P. Zhang. Adaptive force tracking in impedance control. Robot, vol. 21, no. 1, pp. 23-29, 1999.

[28] H. Lee, G. Lee, C. Kwon, N. Noguchi, N. Y. Chong. Switched observer based impedance control for an assistive robotic cart under unknown parameters. In Proceedings of 2012 IEEE RO-MAN, IEEE, Paris, pp. 101-106, 2012.

[29] Z. Su, J. A. Fishel, T. Yamamoto, G. E. Loeb. Use of tactile feedback to control exploratory movements to characterize object compliance. Frontiers in Neurorobotics, vol. 6, pp. 7, 2012.

[30] R. Krten. Getting Started with QNX4: A Guide for Realtime Programmers, Kanata: PARSE Software Devices, 1998

[31] K. H. Yang, W. S. Yu, X. Q. Ji. Rotation estimation for mobile robot based on single-axis gyroscope and monocular camera. International Journal of Automation and Computing, vol. 9, no. 3, pp. 292-298, 2012.

[32] M. M. Zirkohi, M. M. Fateh, M. A. Shoorehdeli. Type-2 fuzzy control for a flexible-joint robot using voltage control strategy. International Journal of Automation and Computing, vol. 10, no. 3, pp. 242-255, 2013. 


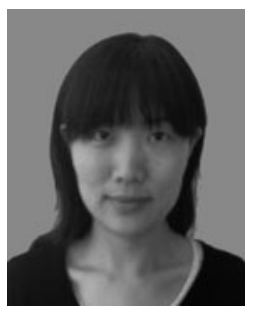

Shuang Gu received her B. Eng. degree in automation control and engineering and M. Sc. degree in control theory and control engineering from Shenyang Jianzhu University, China in 2007 and 2010, respectively. She is currently a Ph. D. candidate in Computer Science and Technology Department at the University of Bedfordshire, UK.

Her research interests include robotics and wireless sensor networks.

E-mail: shuang.gu@beds.ac.uk (Corresponding author)

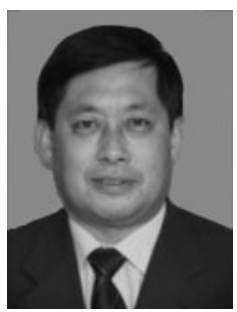

Cheng-Dong Wu received a B. Sc. in electrical automation from University of Shenyang Architectural and Civil Engineering, China in 1983, an M. Sc. in automatic control theory and application from Tsinghua University, China in 1988 and a $\mathrm{Ph}$. D. degree in industrial automation from Northeastern University, China in 1994. Currently, he is a professor in Northeastern University, China. He has published eight books and over 300 journal and conference papers.

His research interests include robotics, wireless sensor networks and image processing.

E-mail: wuchengdong@ise.neu.edu.cn

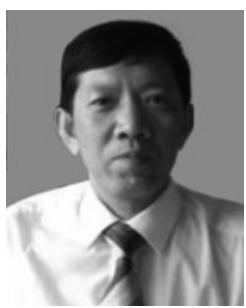

Yong Yue received a B.Sc. in mechanical engineering from the Northeastern University, China and a Ph. D. degree in CAD/CAM from Heriot-Watt University, UK. He is a professor and head of Department of Computer Science and Software Engineering, $\mathrm{Xi}^{\prime}$ an Jiaotong-Liverpool University, China, and a professor at the University of Bedfordshire, UK. He has worked in academia for over 20 years following his 8 years of Industrial experience. He has over 150 research refereed publications.

His research interests include CAD/CAM, computer graphics, robotics, operations research, and artificial intelligence (AI) applications.

E-mail: yong.yue@xjtlu.edu.cn

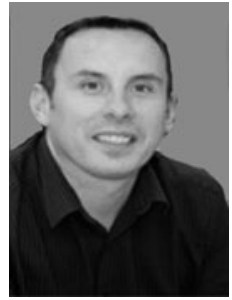

Carsten Maple is Pro-Vice-Chancellor Research and Enterprise, University of Bedfordshire, UK. He graduated with a B.Sc. degree in mathematics and a $\mathrm{Ph}$. D. degree in numerical analysis from the University of Leicester. He is a fellow of the British Computer Society (FBCS) and a Chartered IT Practitioner (CITP). He has published over 150 papers internationally.

His research interests include distributed computing, security, cyberstalking and robotics.

E-mail: carsten.maple@beds.ac.uk

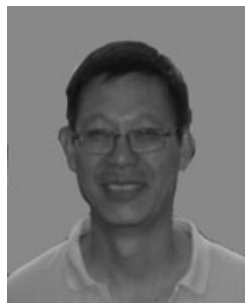

Da-You Li received his B. Eng. and M.Sc. degrees in railway transportation and automation from Beijing Jiaotong University, China in 1982 and 1985, respectively, and his Ph.D. degree in fuzzy backward reasoning control from Cardiff University, Cardiff, UK in 1999. He is the reader of AI \& Robotics at the Department of Computer Science and Technology, University of Bedfordshire, UK.

His research interests include cognitive robotics, multi-agent systems and fuzzy logic and its engineering applications.

E-mail: dayou.li@beds.ac.uk

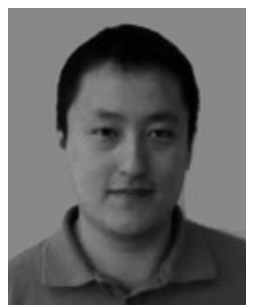

Bei-Sheng Liu is a Ph. D. candidate in the Computer Science and Technology Department at the University of Bedfordshire, UK. He received his undergraduate degree in electronic science and technology from Beijing Jiaotong University, China in 2006.

His research interests include learning and symbolic grounding in robotic systems. E-mail: beisheng.liu@beds.ac.uk 